\title{
Lymphoma in 'India' at the Dawn of Targeted Therapies
}

\author{
Rajan Kapoor $^{1}$ (D) Rajiv Kumar $^{2}$
}

Received: 23 May 2018/Accepted: 5 June 2018/Published online: 18 June 2018

(C) Indian Society of Hematology and Blood Transfusion 2018

Thomas Hodgkin, in 1832, as the 'Inspector of the Dead and Curator of the Museum' at the medical school of Guys hospital published a paper on "On Some Morbid Appearances of the Absorbant Glands and the Spleen", what later on Samuel Wilks in the true spirit of peer recognition named as 'Hodgkin's disease' in 1865 [1]. Hodgkin tried cascarilla, soda and iodine to treat his initial 07 patients with obvious lack of success and who went on to become the subjects of autopsy. The long journey in the management of lymphomas to the current day success story has been phenomenal. We are beginning to look at a chemotherapy and radiotherapy free treatment protocol as the cobweb of signal transduction pathways becomes clearer and we are able to unravel the ways to use the targeted therapies in various combinations to achieve the goal of 'cure'.

While Hodgkins lymphoma achieved much success with the introduction of ABVD in 1970s [2], the heterogenous Non Hodgkin lymphoma (NHL) group has lagged behind with the backbone R-CHOP giving moderate results. Intensification of chemotherapy with protocols like Hyper CVAD, DA-EPOCH-R, etc., have improved the landscape of chemotherapy. However, the long-term effects of chemoradiotherapy has been a major hurdle in increasing the long-term survival of these patients. The increasing understanding of the pathobiology of the various lymphomas, their pathway dependencies and tumor-host

Rajan Kapoor

majrkapoor@gmail.com

1 Department of Hematology, Army Hospital R\&R, New Delhi, India

2 Department of Hematology, Indian Naval Hospital Ship Asvini, Mumbai, India interface, has provided new opportunities to use this knowledge to generate targeted therapies.

The introduction of monoclonal antibodies and the success of Rituximab was a major breakthrough in improving the survival of patients with CD20 + NHL [3]. Similarly anti CD30 Brentuximab Vedotin (BV) became a major success story in relapse-refractory Hodgkins disease. $\mathrm{BV}$ now threatens to replace the conventional Bleomycin, "B" of ABVD, a drug attributed to the most sinister long term pulmonary toxicity of ABVD, as a first line therapy [4].

Moving ahead from surface targets, the pathway dependencies of lymphomas were extensively studied and lead to the approval of Ibrutinib, a BTK inhibitor and a first drug of its kind, for Chronic Lymphocytic Leukemia with del17p. This success story has opened the pandora's box for targeted therapies with various drugs like Idelalisib, venetoclax, panabinostat, etc., providing good results in relapse refractory cases, which were considered incurable.

The introduction of tongue-twister CAR-T cell therapies like axicabtagene ciloleucel and tisagenlecleucel has brought Immunotherapy to the forefront of management of lymphoproliferative disorders. PD-L1 inhibitors like Nivolumab and Pembrolizumab can be considered as a major breakthrough in our understanding of the tumorimmunity cross talk and the benefits of their exploitation in treating these malignancies.

The rapid strides in this field comes with its challenges of a continuously changing landscape of management guidelines. Anyone who is dealing with the management of lymphomas in India finds it difficult to remain abreast with the latest in this field. The need for an India-specific guideline may be partially fulfilled by the updated consensus statement presented in the current issue of IJHBT [5]. The consensus document on the management of 
Lymphoma from an Indian Expert Group is a commendable effort to bridge this gap and provide the details on a single platform. Large number of 'Indian' lymphoma experts have come together and brought out a useful resource for all hemato-oncologists which includes all aspects of lymphoma from diagnosis, staging to treatment strategies in a readable form. Addition of resource stratified diagnostic workup in a tabular form is especially important in country like India where income disparity is so huge that "one size fits all" is not possible. There are 26 tables in the document which serve as a ready reckoner for anyone referring to these guidelines. However, those who have been newly initiated to the field of Lymphoma may find the "HL/NHL/CLL" mix in a single document a bit difficult to assimilate.

The road ahead of Indian 'Lymphoma-doctors' is a difficult one. Though there are few epidemiologic studies from India, there is very little credible data emerging from the sub-continent and direct extrapolation of the western data on our population is not desirable. Original research requires institutional funding and a major change in the way we look at research in our country. The way forward lies in good inter-institutional collaboration's and not in creating small isolated islands of expertise/excellence. There are also major challenges in the access to these therapies owing to the high costs involved. Financial models catering to different sections of the society with active Governmental and Non-Governmental/Insurance participation is the need of the hour. Scientific knowledge will be of little use if the benefits of these path-breaking research is not passed on to the large number of patients suffering in every corner of our country.

\section{Compliance with Ethical Standards}

Conflict of interest The authors declare that they have no conflict of interest.

Ethical Approval This article does not contain any studies with human participants or animals performed by any of the authors.

\section{References}

1. Bonnadonna G (2000) Historical review of Hodgkin's disease. Br J Haematol 110:504-511

2. Bonnadonna G, Zucali R, Monfardini S et al (1975) Combination chemotherapy of Hodgkin's disease with adriamycin, bleomycin, vinblastine, and imidazole carboxamide versus MOPP. Cancer $36: 252-259$

3. Maloney DG, Grillo-Lopez AJ, White CA et al (1997) IDECC2B8 (Rituximab) anti-CD20 monoclonal antibody therapy in patients with relapsed low-grade non-Hodgkin's lymphoma. Blood 90:2188-2195

4. Stephens DM (2017) Brentuximab: Is it time for for a new "B" in ABVD? Blood 130:1281-128

5. Kakroo A, Bafna A, Gogia A, Vora A, Pathak A, Korula A et al (2018) Management of Lymphomas: consensus document 2018 by an Indian Expert Group. Indian J Hematol Blood Transfus (Online First) 